\title{
Ultrasonically enhanced rifampin activity against internalized Staphylococcus aureus
}

\author{
SI-FENG SHI, XIAN-LONG ZHANG, CHEN ZHU, DE-SHENG CHEN and YONG-YUAN GUO \\ Department of Orthopaedic Surgery, Shanghai Sixth People's Hospital, \\ Shanghai Jiao Tong University School of Medicine, Shanghai 200233, P.R. China
}

Received July 23, 2012; Accepted October 12, 2012

DOI: $10.3892 /$ etm.2012.758

\begin{abstract}
Staphylococcus aureus (S. aureus) is the principle causative agent of osteomyelitis, accounting for $80 \%$ of all human cases. S. aureus internalized in osteoblasts escapes immune response, including engulfment by phagocytes. It also escapes the action of a number of antibiotics. Ultrasound increases cell membrane permeability to a number of drugs. Following an internalization assay, we used low-frequency, low-power ultrasound combined with the antibiotic rifampin to target $S$. aureus internalized in human osteoblasts. Tryptic soy agar (TSA) was used to quantitate the antibacterial effect of rifampin combined with low-frequency ultrasound. A Cell Counting Kit-8 (CCK-8) assay was used to evaluate cell viability following exposure to ultrasound. Our data revealed that rifampin successfully penetrates into osteoblasts and kills internalized $S$. aureus in osteoblasts, while low-frequency ultrasound promotes this process. Ultrasound had a negative impact on the cell viability of osteoblasts; however, this damage was slight and reversible. Ultrasound-enhanced antibiotic efficiency to bacteria internalized in the osteoblasts may contribute to the control of chronic infection to reduce recurrence.
\end{abstract}

\section{Introduction}

Osteomyelitis is an inoculation of bacteria into bone as a result of hematogenous seeding, surgical contamination, spread of infection from an adjacent area, trauma coinciding with contamination or injury to a limb without the protective soft tissue envelope (1). The implantation of foreign bodies increases the likelihood of infection. In this disease, bone is colonized with microorganisms, with associated inflamma-

Correspondence to: Professor Xian-long Zhang, Department of Orthopaedic Surgery, Shanghai Sixth People's Hospital, Shanghai Jiao Tong University School of Medicine, 600 Yi Shan Road, Xu Hui District, Shanghai 200233, P.R. China

E-mail: zhangxianlong20101@163.com

Key words: ultrasound, osteoblast, Staphylococcus aureus, rifampin tion and bone destruction (2). Chronic infection is difficult to eradicate. Aggressive surgical debridement of the infected or necrotic tissue and extensive bone and soft tissue reconstruction are usually required to cure the disease (1). Foreign bodies need to be removed in the majority of cases in order to eradicate infection. A small proportion of seriously ill patients require amputation. Besides surgical treatment, intravenous antibiotics for extended periods of time is another form of treatment for osteomyelitis. However, due to the locally compromised blood supply, a therapeutic level of antibiotics is rarely achieved $(1,3)$.

The gram-positive organism Staphylococcus aureus (S. aureus) is the principle causative agent of osteomyelitis, accounting for $80 \%$ of all human cases $(4,5)$. Adhesion molecules of $S$. aureus facilitate its binding to the bone matrix. Toxin secretion such as interleukin (IL)-1, IL-6 and tumor necrosis factor (TNF)- $\alpha$ may produce $S$. aureus-induced osteomyelitis and stimulate bone resorption (6). The bacterial biofilm is considered to be the main reason for refractoriness of osteomyelitis. However, previous research demonstrated that $S$. aureus not only colonized bone matrix, but also invaded osteoblasts (2,5,7-9). This phenomenon has been demonstrated in vitro and in vivo (10). The capability of $S$. aureus to invade and survive within osteoblasts may be an important reason as to why chronic osteomyelitis is difficult to be eradicate. S. aureus internalized in osteoblasts avoids immune responses, including engulfment by phagocytes, as well as the action of many forms of antibiotics. S. aureus survived in the intracellular environment of osteoblasts and maintained the vitality to invade other osteoblasts $(1,11)$. This may explain the recurrence and chronic course of this disease (3).

Numerous types of antibiotics have been employed in the treatment of osteomyelitis, including gentamicin, cephalosporins, vancomycin, clindamycin and rifampin. However, the majority of these antibiotics cannot penetrate eukaryotic cells. Rifampin is a hydrophobic antibiotic and is able to penetrate cell membranes and enter osteoblasts. A study in vitro demonstrated its bactericidal effect in the intracellular environment (3). In clinical practice, rifampin also demonstrates satisfactory results in the treatment of osteomyelitis, particularly in prosthesis infections (12-15). Other antibiotics capable of penetrating eukaryotic cells, including clindamycin, also have antibacterial effects in the intracellular environment (3). 
In order to kill bacteria internalized in the osteoblasts, it is necessary to increase the intracellular concentration of antibiotics. The hydrophobic properties of the drugs and the permeability of cell membranes are two important factors when determining the concentration of a drug in the intracellular environment. The aim of the present study was to improve the permeability of the osteoblast membrane in order to increase the concentration of antibiotics to kill S. aureus in the intracellular environment.

The application of ultrasound as an adjunct has showed satisfactory results in the treatment of tumors, infection and DNA transfection in in vitro and in vivo research. It increases the permeability of eukaryotic and prokaryotic cells to drugs, DNA and nanoparticles. Previous research has documented that ultrasound enhances the activity of a number of antibiotics against certain bacteria in plankton and in biofilms $(16,17)$. Additionally, further research revealed that ultrasound allows antibiotics to transport through the biofilm more easily and therefore increases the drug concentration surrounding the bacteria (18). Ultrasound perturbs the bacteria cell membrane, rendering it more permeable to antibiotics. Ultrasound-enhanced antibiotic efficacy has also been demonstrated in planktonic bacteria. However, no research has reported on the ultrasonic adjuvant effect to bacteria in the intracellular environment. The present study tests the hypothesis that low frequency, low-power ultrasound enhances the antibiotic (rifampin) effect of $S$. aureus internalized in human osteoblasts (19).

\section{Materials and methods}

Bacterial strains, media and antibiotics. Cultures of S. aureus (ATCC12598) were grown on nutrient agar plates. Tryptic soy broth (TSB; Difco, Detroit, MI, USA) was inoculated with one colony from the plate and a culture was grown overnight at $37^{\circ} \mathrm{C}$ with agitation to the plate.

Cell culture. Normal human osteoblasts (sv 40) were incubated at $37^{\circ} \mathrm{C}$ under a humidified atmosphere containing $5 \% \mathrm{CO}_{2}$ in Dulbecco's modified Eagle's medium (DMEM; Gibco, Beijing,China) supplemented with $10 \%$ (v/v) fetal bovine serum. Once the cells had reached $\sim 80 \%$ confluence, they were removed from the flasks using $0.025 \%$ trypsin and $0.01 \%$ ethylenediaminetetraacetic acid (EDTA) in phosphatebuffered saline (PBS). The growth medium was changed every $48 \mathrm{~h}$ after seeding. The study was approved by the ethics committee of the Department of Orthopaedics, The Sixth Affiliated People's Hospital, Shanghai Jiao Tong University School of Medicine, (Shanghai, China).

Internalization assay. S. aureus was grown overnight (16 h) in $5 \mathrm{ml} \mathrm{TSB}$ in a water bath at $37^{\circ} \mathrm{C}$ with agitation. The bacteria were harvested by centrifugation for $5 \mathrm{~min}$ at $5,000 \mathrm{rpm}$ at $4^{\circ} \mathrm{C}$ and washed twice in $5 \mathrm{ml}$ PBS. The pellet was then resuspended in $5 \mathrm{ml}$ osteoblast growing medium (OBGM) without antibiotics. Confluent cell layers of osteoblasts were washed three times with $5 \mathrm{ml}$ PBS to remove growth media containing antibiotics. Osteoblasts were then infected at a multiplicity of infection (MOI) of 200:1 with S. aureus. After infection for $45 \mathrm{~min}$ at $37^{\circ} \mathrm{C}$, cell cultures were washed and then incubated with OBGM containing $100 \mu \mathrm{g} / \mathrm{ml}$ gentamicin for $4 \mathrm{~h}$ to kill the remaining extracellular $S$. aureus. Gentamicin cannot penetrate normal eukaryotic cells, so at this time, only intracellular bacteria remain.

Ultrasound treatment combined with antibiotic activity. Non-focused ultrasonic transducers were used in this experiment to deliver the ultrasound. A function generator (XinZhi, Biotechnology Co., Ningbo, China) created a non-continuous wave at a frequency of $20 \mathrm{kHz}$. The osteoblasts were divided into 4 groups and treated as follows: in group A, osteoblasts with OBGM containing $30 \mu \mathrm{g} / \mathrm{ml}$ of rifampin were exposed to ultrasound for 60 cycles of $5 \mathrm{sec}$ ultrasound with $2 \mathrm{sec}$ intervals $(200 \mathrm{~W})$. In group B, osteoblasts were exposed to rifampin without ultrasound. In group $\mathrm{C}$, the osteoblasts were exposed to ultrasound with OBGM not containing rifampin and rifampin was added to OBGM $30 \mathrm{~min}$ after exposure to ultrasound. In group D, the osteoblasts were not exposed to ultrasound.

Assay to determine rifampin antibiotic activity combined with ultrasound. After rifampin addition and ultrasound intervention (6 h later), osteoblast cultures were washed with PBS 3 times and subsequently lysed by the addition of $1 \mathrm{ml} 0.2 \%$ Triton X-100 (Fisher Biotech, Fair Lawn, NJ, USA), followed by standard serial dilution, plating on tryptic soy agar (TSA), incubation at $37^{\circ} \mathrm{C}$ overnight and enumeration of resulting colony forming units.

Osteoblast viability following ultrasound exposure (CCK-8). Human osteoblasts (sv40) were inoculated in a 96-well plate. Once the cells had reached $\sim 80 \%$ confluence, they were exposed to ultrasound as previously described. The osteoblasts were then cultured in standard environment for another 24 h. The Cell Counting Kit-8 (CCK-8; Beyotime, Shanghai, China) was employed to quantitatively evaluate osteoblast viability (20). Briefly, the culture medium was removed and the cultures were washed with PBS twice. Serum-free DMEM $(\sim 100 \mu \mathrm{l})$ and CCK-8 (10 $\mu \mathrm{l})$ solution were added to each well. Following incubation at $37^{\circ} \mathrm{C}$ for $2 \mathrm{~h}$, a microplate reader (BioTek Instruments, Winooski, VT, USA) was employed to determine the optical density (OD) at $450 \mathrm{~nm}$ and compared with the control group. Three duplicate experiments were performed to assess the cell viability. The following equation was used: cell viability $=\left(\mathrm{OD}_{\text {sample }} / \mathrm{OD}_{\text {control }}\right) \times 100$.

Statistical analysis. Each experiment was performed three to six times and the quantitative results were expressed as mean \pm standard deviation. Differences between the means were analyzed by using independent $t$-test. $P<0.05$ was considered to indicate a statistically significant difference.

\section{Results}

Internalization of $S$. aureus. We quantified the invasion of $S$. aureus into osteoblasts using TSA plates. Following an internalization assay, the osteoblast cultures were washed three times and subsequently lysed by the addition of $1.2 \mathrm{ml}$ $0.1 \%$ Triton X-100. Suspension dilutions of the lysates were plated in triplicate on TSA plates followed by incubation at 


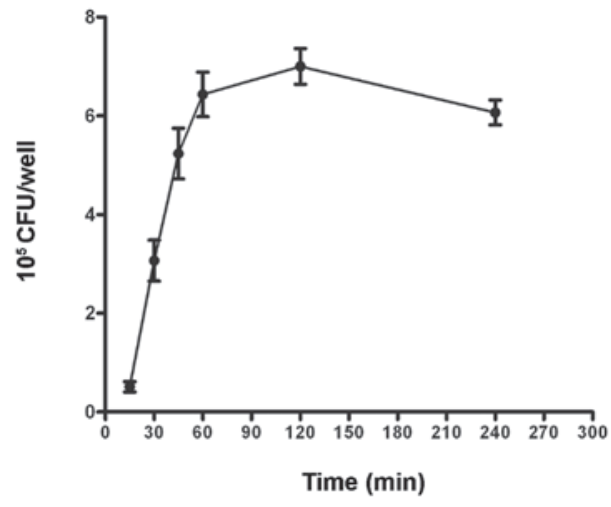

Figure 1. Quantification of internalized S. aureus in human osteoblasts. The sv40 human osteoblasts were exposed to S. aureus ATCC12598 at an MOI of 200 for the indicated time. Gentamicin was used to kill the remaining extracellular $S$. aureus for $4 \mathrm{~h}$. The osteoblasts were lysed by the addition of $1 \mathrm{ml}$ $0.2 \%$ Triton $\mathrm{X}-100$, followed by standard serial dilution, plating on tryptic soy agar, incubation at $37^{\circ} \mathrm{C}$ overnight and enumeration of resulting colony forming units. CFU, colony forming unit; MOI, multiplicity of infection.

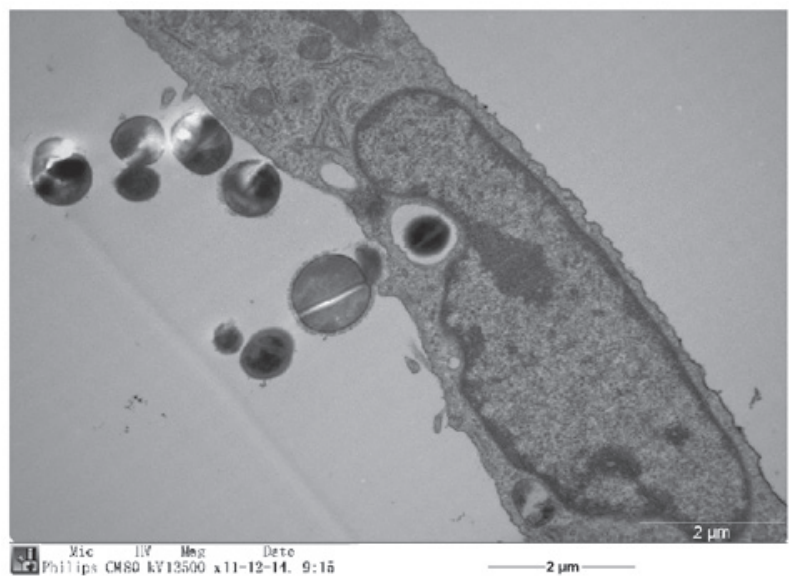

Figure 2. Internalization of S. aureus by an osteoblast observed under transmission electron microscopy.

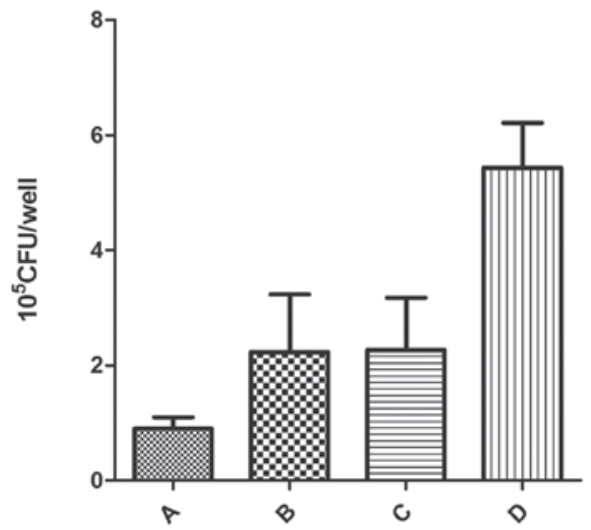

Figure 3. Quantification of internalized S. aureus in human osteoblasts following exposure to ultrasound and rifampin. (A) Osteoblasts with OBGM containing $30 \mathrm{mg} / \mathrm{ml}$ of rifampin were exposed to ultrasound; (B) osteoblasts were exposed to rifampin without ultrasound; (C) osteoblasts were exposed to ultrasound with OBGM not containing rifampin and rifampin was added to OBGM 30 min after exposure to ultrasound; (D) osteoblasts were not exposed to ultrasound or rifampin. CFU, colony forming unit; OBGM, osteoblast growing medium.

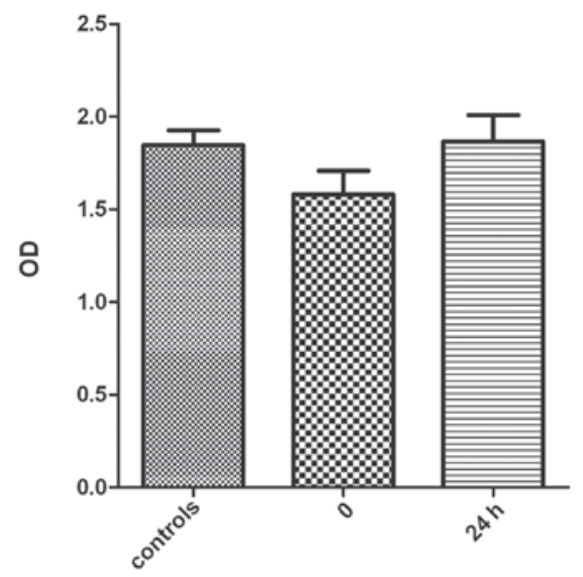

Figure 4. Comparison of osteoblast viability immediately after exposure to ultrasound, $24 \mathrm{~h}$ after exposure to ultrasound and the control group without exposure to ultrasound. OD, optical density.

$37^{\circ} \mathrm{C}$ overnight. Results showed that the invasion of $S$. aureus increased in a time-dependent manner following infection at an MOI of 200 (Fig. 1). Internalization of S. aureus by osteoblasts was confirmed by transmission electron microscopy (Fig. 2).

Effect of ultrasound combined with antibiotic inhibition of S. aureus internalized in osteoblasts. When $S$. aureus cells were exposed to antibiotics combined with ultrasound, the number of viable organisms in the intracellular environment of osteoblasts decreased. Rifampin, a bactericidal nucleic acid synthesis inhibitor, is readily able to penetrate eukaryotic cells. The number of viable $S$. aureus cells within osteoblasts not treated with ultrasound was smaller compared with the control group, but larger compared with that of rifampin combined with ultrasound. There was no significant difference between group B and group C. These results suggest that rifampin successfully penetrates osteoblasts and kills $S$. aureus internalized in the osteoblasts, while ultrasound promotes this process (Fig. 3).

Osteoblast viability following ultrasound exposure. The CCK-8 assay was employed to compare viability of sv40 osteoblasts following exposure to ultrasound with normal cells. The results revealed a decreased viability of the osteoblasts immediately after exposure to ultrasound. However, the viability of osteoblasts exposed to ultrasound increased after being cultured in the standard atmosphere for $24 \mathrm{~h}$. There was no significant difference in viability of osteoblasts $24 \mathrm{~h}$ after exposure to ultrasound and the control group (Fig. 4). These results suggest that low-frequency ultrasound has a negative impact on cell viability of osteoblast sv40; however, this damage is slight and reversible.

\section{Discussion}

Ultrasound has been used in a number of applications in medicine and research $(16,18,21,22)$. Previous research has shown that low-frequency ultrasound, when combined with antibiotics, significantly enhances the bactericidal action 
of antibiotics $(16,22,23)$. Previous research has shown that ultrasound effectively enhances the antibacterial effect of certain antibiotics against a culture of bacteria in in vitro and in vivo biofilms $(17,23)$. Ultrasound increases the transport of antibiotics through biofilms, which is a barrier for antibiotics to penetrate in order to kill bacteria inside (18). Carmen et al demonstrated that ultrasonication significantly increases the transport of gentamicin through the biofilm. Insonation of biofilms for $45 \mathrm{~min}$ more than doubles the gentamicin concentration compared to the non-insonated counterparts. This enhanced transport may be partially responsible for the increased killing of biofilm bacteria exposed to combinations of antibiotics and ultrasound (18). Ultrasound-enhanced antibiotic efficacy has also be conducted in in vivo research. Carmen et al investigated the combination of low frequency ultrasound and vancomycin in treating Staphylococcus epidermidis infections in a rabbit model and the result showed enhanced vancomycin activity against $S$. epidermidis biofilms. At $48 \mathrm{~h}$ of insonation there were significantly fewer viable bacteria in the insonated biofilm (17).

However, ultrasound-enhanced antibiotic activity in planktonic cultures with no extensive exopolymer matrix to hinder antibiotic transport, indicates that there must be other mechanisms involved in the ultrasound-enhanced antibiotic effect on organisms. Ultrasound is postulated to increase antibiotic concentration inside cells by rendering the cell membrane more permeable to the antibiotic (19). Perturbation caused by ultrasound results in decreased stability of the phospholipid bilayer. Rapoport et al observed that the passage of stearic acid (lipophilic substances) into the outer lipid bilayer was ultrasonically enhanced in Pseudomonas aeruginosa $(24,25)$.

A similar reversible destabilization may occur in eukaryotic cell membranes. Studies have indicated that lowfrequency ultrasound destabilizes lipid layers in the skin, thus enhancing the permeability of drugs $(16,26)$. It has also been demonstrated that plasmid DNA was effectively delivered into Saccharomyces cerevisiae by using low-frequency ultrasound $(16,27)$. Our current study indicates that ultrasound may create perturbations in the outer membrane lipid bilayer of osteoblasts sufficiently large for the passage of rifampin.

Recently, research into ultrasound-aided cancer chemotherapy has been conducted to enhance the overall cytotoxic effect of the therapeutic agent with no apparent hyperthermic event (28). Sonoporation is an ultrasound-induced means of increasing the permeability of cell membranes to promote the passage of chemotherapy agents across the cell membrane, thereby facilitating entry of the relevant drug into the target cell (29-31). Our results are consistent with the previous result. Rifampin is a lipophilic drug and the ultrasonically-enhanced bactericidal capacity in the intracellular environment may be due to the increased drug concentration in the intracellular environment (19).

Sonoporation has shown promising prospects (32). Although the mechanism by which ultrasound enhances antibiotic action is not fully known, it may be due to perturbation of the cell membrane or stress responses by the bacteria $(22,33)$. Sonoporation enhances cell membrane permeability allowing transfer of macromolecules. Ultrasound-induced biological effects are commonly considered to be caused by acoustic cavitation, which may collapse and lead to the induction of tran- sient holes in the cell membrane. It has been hypothesized that antibiotics are transferred into cells across the cell membrane via ultrasound-induced pores. This is a transient phenomenon and our results also give indirect evidence of this, as the cell viability decreased immediately after the application of ultrasound, but recovered $24 \mathrm{~h}$ later (19). Ultrasound-induced pores have also been confirmed by scanning election microscopy (SEM) observations, although the size distribution could not be defined (32).

Ultrasonic waves pass directly through cells with little absorption or scattering. The pressure oscillations of ultrasound produces gas bubbles ranging in size from approximately 1 to $100 \mu \mathrm{m}$ in diameter in the liquid. The oscillations of bubbles, also called cavitation, are generally divided into 'stable' and 'collapse' types. Both types of cavitation are reported to increase membrane permeability in eukaryotic cells (19). Stable cavitation is the low intensity oscillation of the bubbles without complete collapse and collapse cavitation occurs at higher intensity levels.

There is an intensity threshold for the production of collapse cavitation and collapse cavitation is absent below this threshold; however, stable cavitation occurs readily $(19,34)$. The lower shear forces caused by stable cavitation may also be sufficiently stressful to perturb the outer membrane (35). Ultrasound has been shown to enhance fluorescent probe uptake in corneal endothelium and various cancer cells, as well as allow penetration of Ara-C into HL-60 cells and allow transfection of eukaryotes with plasmid DNA $(19,28,32,36,37)$. When these bubbles collapse, they violently accelerate the fluid around them, producing a high temperature and free radicals as well as generating high liquid shear force. Cell membranes may become stressed or damaged by shear force, heat or free radicals (38). Stress and high velocity jet of liquid toward the cell membrane during bubble expansion may contribute to the cell membranes damage $(19,39)$.

Although ultrasound is used as a means to lyse bacteria, the low-frequency and lower intensities of ultrasound did not directly lyse the bacterial cells or permanently damage the outer bacterial membrane (19). Indeed, ultrasound at the intensities used in our research did not directly reduce viability of bacteria. In the present study, group C, with ultrasound in the absence of antibiotics, demonstrated no significant decrease in the number of bacteria (Fig. 3). In fact, in certain situations, lowfrequency ultrasound increased bacteria cell growth (38). Pitt et al used ultrasound to irradiate bacterial cells (S. epidermidis, P. aeruginosa and Escherichia coli cells) attached to polyethylene surfaces. They found that low frequency ultrasound $(70 \mathrm{kHz})$ of low acoustic intensity increased the growth rate of the cells compared to growth without ultrasound. Ultrasound has also demonstrated the ability to enhance planktonic growth of S. epidermidis and other planktonic bacteria. These research results may be due to an increased rate of transport of oxygen and nutrients to the cells and waste products away from the cells by ultrasound (38).

Ultrasound increases the permeability of cells without killing them, to allow drugs to enter the cells (34). A major challenge to the application of sonoporation in drug delivery is to optimize the ultrasound parameters to increase the permeability of cell membranes and to maintain good cell viability (40). Acoustic cavitation induced by ultrasound increases cell permeability and 
facilitates drug internalization in the cells. However, it may also damage or kill the cells if the pores induced by cavitation are too large such that the cell membrane cannot reseal quickly. For large molecules, this is a more serious problem since formation of larger pores is needed on the cell surface, risking disruption to the cell membrane, loss of vital cytoplasmic compounds and resulting in cell death. The results presented in this study show that a reasonable result can be achieved under the correct ultrasound conditions. The cell viability is as high as $80 \%$ compared with the control group immediately after sonoporation. However, this is a transient process, as shown in Fig. 4, since cell viability returns $24 \mathrm{~h}$ after ultrasound exposure.

The use of ultrasound to transfect eukaryotes with plasmid DNA indicates that the enhanced plasma membrane permeability is transient and the cell membrane can seal or heal to preserve viability $(19,32)$. Mitragotri et al used low-frequency ultrasound to destabilize lipid layers and enhance permeability of drugs in the skin. Following ultrasonic treatment, the original permeability was eventually restored (26). Freeze-fracture electron microscopy has shown that, a few seconds after the electric pulse, the pores began to reseal; $5 \mathrm{sec}$ post-electroporation, the majority of the pores became shallow and smaller, while at $10 \mathrm{sec}$ the pore-like structures on the cell membrane had almost disappeared $(32,41)$. Guzman et al reported similar results when cells were exposed to low frequency ultrasound $(24 \mathrm{kHz})(37,43,44)$. Uptake of bovine serum albumin and calcein was completely abolished when the compounds were added 1 and 2 min after ultrasound exposure, respectively (18). The short duration of membrane pore opening implies that, if the drug is to be effectively internalized, it should be close to the membrane when poration occurs $(32,42-44)$. Therefore, application of drugs and the interaction between drugs and cells should be performed prior to sonication.

Chronic orthopedic infection is a serious concern in clinical practice. Ultrasound-enhanced antibiotic efficiency to the bacteria internalized in the osteoblast may contribute to the control of chronic infection and reduce the recurrence. Further research is required to allow for better understanding of these fundamental issues and to fully exploit the potential use of sonoporation in treatment of infections.

\section{Acknowledgements}

This study was financially supported by the Characteristic Construction Foundation of the Sixth People's Hospital affiliated to Shanghai Jiaotong University.

\section{References}

1. Ellington JK, Harris M, Webb L, et al: Intracellular Staphylococcus aureus. A mechanism for the indolence of osteomyelitis. J Bone Joint Surg Br 85: 918-921, 2003.

2. Wright JA and Nair SP: Interaction of staphylococci with bone. Int J Med Microbiol 300: 193-204, 2010.

3. Ellington JK, Harris M, Hudson MC, Vishin S, Webb LX and Sherertz R: Intracellular Staphylococcus aureus and antibiotic resistance: implications for treatment of staphylococcal osteomyelitis. J Orthop Res 24: 87-93, 2006.

4. Ning R, Zhang X, Guo X and Li Q: Attachment of Staphylococcus aureus is required for activation of nuclear factor kappa B in human osteoblasts. Acta Biochim Biophys Sin 42: 883-892, 2010.

5. Chihara S and Segreti J: Osteomyelitis. Disease-a-Month 56: 6-31, 2010.
6. Ishida I, Kohda C, Yanagawa Y, Miyaoka H and Shimamura T: Epigallocatechin gallate suppresses expression of receptor activator of NF-kappaB ligand (RANKL) in Staphylococcus aureus infection in osteoblast-like NRG cells. J Med Microbiol 56: 1042-1046, 2007.

7. Jin C and Flavell RA: Molecular mechanism of NLRP3 inflammasome activation. J Clin Immunol 30: 628-631, 2010.

8. Chauhan VS and Marriott I: Differential roles for NOD2 in osteoblast inflammatory immune responses to bacterial pathogens of bone tissue. J Med Microbiol 59: 755-762, 2010.

9. Webb LX, Wagner W, Carroll D, Tyler H, Coldren F and Martin E: Osteomyelitis and intraosteoblastic Staphylococcus aureus. J Surg Orthop Adv 16: 73-78, 2007.

10. Reilly SS: In vivo internalization of Staphylococcus aureus by embryonic chick osteoblasts. Bone 26: 63-70, 2000.

11. Shi S and Zhang X: Interaction of Staphylococcus aureus with osteoblasts (Review). Exp Ther Med 3: 367-370, 2012.

12. Lefebvre M, Jacqueline C, Amador G, et al: Efficacy of daptomycin combined with rifampicin for the treatment of experimental meticillin-resistant Staphylococcus aureus (MRSA) acute osteomyelitis. Int J Antimicrob Agents 36: 542-544, 2010.

13. Khanlari B, Elzi L, Estermann L, et al: A rifampicin-containing antibiotic treatment improves outcome of staphylococcal deep sternal wound infections. J Antimicrob Chemother 65: 1799-1806, 2010.

14. Garrigos C, Murillo O, Euba G, et al: Efficacy of usual and high doses of daptomycin in combination with rifampin versus alternative therapies in experimental foreign-body infection by methicillin-resistant Staphylococcus aureus. Antimicrob Agents Chemother 54: 5251-5256, 2010.

15. El Helou OC, Berbari EF, Lahr BD, et al: Efficacy and safety of rifampin containing regimen for staphylococcal prosthetic joint infections treated with debridement and retention. Eur J Clin Microbiol Infect Dis 29: 961-967, 2010.

16. Rediske AM, Hymas WC, Wilkinson R and Pitt WG: Ultrasonic enhancement of antibiotic action on several species of bacteria. J Gen Appl Microbiol 44: 283-288, 1998.

17. Carmen JC, Roeder BL, Nelson JL, et al: Ultrasonically enhanced vancomycin activity against Staphylococcus epidermidis biofilms in vivo. J Biomater Appl 18: 237-245, 2004.

18. Carmen JC, Nelson JL, Beckstead BL, et al: Ultrasonic-enhanced gentamicin transport through colony biofilms of Pseudomonas aeruginosa and Escherichia coli. J Infect Chemother 10: 193-199, 2004.

19. Runyan CM, Carmen JC, Beckstead BL, Nelson JL, Robison RA and Pitt WG: Low-frequency ultrasound increases outer membrane permeability of Pseudomonas aeruginosa. J Gen Appl Microbiol 25: 295-301, 2006.

20. Wang L, Wang ZH, Shen CY, You ML, Xiao JF and Chen GQ: Differentiation of human bone marrow mesenchymal stem cells grown in terpolyesters of 3-hydroxyalkanoates scaffolds into nerve cells. Biomaterials 31: 1691-1698, 2010.

21. Williams RG and Pitt WG: In vitro response of Escherichia coli to antibiotics and ultrasound at various insonation intensities. J Biomater Appl 12: 20-30, 1997.

22. Rediske AM, Roeder BL, Brown MK, et al: Ultrasonic enhancement of antibiotic action on Escherichia coli biofilms: an in vivo model. Antimicrob Agents Chemother 43: 1211-1214, 1999.

23. Carmen JC, Roeder BL, Nelson JL, et al: Treatment of biofilm infections on implants with low-frequency ultrasound and antibiotics. Am J Infect Control 33: 78-82, 2005.

24. Rapoport N, Smirnov AI, Timoshin A, Pratt AM and Pitt WG: Factors affecting the permeability of Pseudomonas aeruginosa cell walls toward lipophilic compounds: effects of ultrasound and cell age. Arch Biochem Biophys 344: 114-124, 1997.

25. Rediske AM, Rapoport N and Pitt WG: Reducing bacterial resistance to antibiotics with ultrasound. Lett Appl Microbiol 28: 81-84, 1999.

26. Mitragotri S, Blankschtein D and Langer R: Transdermal drug delivery using low-frequency sonophoresis. Pharm Res 13: 411-420, 1996.

27. Wyber JA, Andrews J and D'Emanuele A: The use of sonication for the efficient delivery of plasmid DNA into cells. Pharm Res 14: 750-756, 1997.

28. Tachibana K, Uchida T, Tamura K, Eguchi H, Yamashita N and Ogawa K: Enhanced cytotoxic effect of Ara-C by low intensity ultrasound to HL-60 cells. Cancer Lett 149: 189-194, 2000.

29. Li YS, Reid CN and McHale AP: Enhancing ultrasound-mediated cell membrane permeabilisation (sonoporation) using a high frequency pulse regime and implications for ultrasound-aided cancer chemotherapy. Cancer Lett 266: 156-162, 2008. 
30. Iwanaga $\mathrm{K}$, Tominaga $\mathrm{K}$, Yamamoto $\mathrm{K}$, et al: Local delivery system of cytotoxic agents to tumors by focused sonoporation. Cancer Gene Ther 14: 354-363, 2007.

31. Kinoshita M and Hynynen K: Key factors that affect sonoporation efficiency in in vitro settings: the importance of standing wave in sonoporation. Biochem Biophys Res Commun 359: 860-865, 2007.

32. Mehier-Humbert S, Bettinger T, Yan F and Guy RH: Plasma membrane poration induced by ultrasound exposure: implication for drug delivery. J Control Release 104: 213-222, 2005.

33. Qian Z, Sagers RD and Pitt WG: Investigation of the mechanism of the bioacoustic effect. J Biomed Mater Res 44: 198-205, 1999.

34. Liu J, Lewis TN and Prausnitz MR: Non-invasive assessment and control of ultrasound-mediated membrane permeabilization. Pharm Res 15: 918-924, 1998.

35. Ananta E, Voigt D, Zenker M, Heinz V and Knorr D: Cellular injuries upon exposure of Escherichia coli and Lactobacillus rhamnosus to high-intensity ultrasound. J Appl Microbiol 99: 271-278, 2005.

36. Saito K, Miyake K, McNeil PL, Kato K, Yago K and Sugai N Plasma membrane disruption underlies injury of the corneal endothelium by ultrasound. Exp Eye Res 68: 431-437, 1999.

37. Guzman HR, McNamara AJ, Nguyen DX and Prausnitz MR: Bioeffects caused by changes in acoustic cavitation bubble density and cell concentration: a unified explanation based on cell-to-bubble ratio and blast radius. Ultrasound Med Biol 29: $1211-1222,2003$.
38. Pitt WG and Ross SA: Ultrasound increases the rate of bacterial cell growth. Biotechnol Prog 19: 1038-1044, 2003.

39. Gracewski SM, Miao H and Dalecki D: Ultrasonic excitation of a bubble near a rigid or deformable sphere: implications for ultrasonically induced hemolysis. J Acoust Soc Am 117: 1440-1447, 2005.

40. Huber PE and Pfisterer P: In vitro and in vivo transfection of plasmid DNA in the Dunning prostate tumor R3327-AT1 is enhanced by focused ultrasound. Gene Ther 7: 1516-1525, 2000.

41. Chang DC: Structure and dynamics of electric field-induced membrane pores as revealed by rapid-freezing electron microscopy. In: Guide to Electroporation and Electrofusion. Academic Press, Inc., San Diego, pp9-27, 1992

42. Lee EK, Gallagher RJ, Campbell AM and Prausnitz MR Prediction of ultrasound-mediated disruption of cell membranes using machine learning techniques and statistical analysis of acoustic spectra. IEEE Trans Biomed Eng 51: 82-89, 2004.

43. Guzman HR, Nguyen DX, Khan S and Prausnitz MR: Ultrasoundmediated disruption of cell membranes. II. Heterogeneous effects on cells. J Acoust Soc Am 110: 597-606, 2001.

44. Guzman HR, Nguyen DX, Khan S and Prausnitz MR: Ultrasoundmediated disruption of cell membranes. I. Quantification of molecular uptake and cell viability. J Acoust Soc Am 110: 588-596, 2001. 